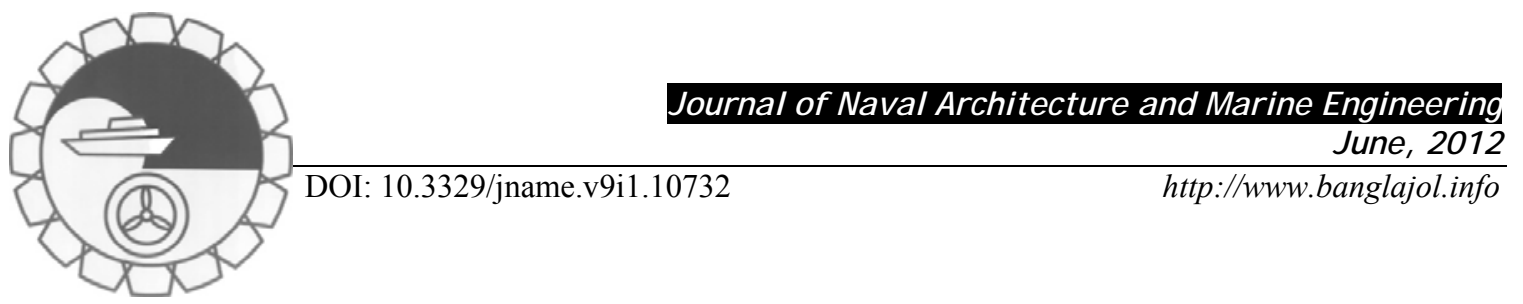

\title{
STUDY OF MOTION OF SPAR-TYPE FLOATING WINDTURBINES IN WAVES WTH EFFECT OF GYRO MOMENT AT INCLINATION
}

\section{N. Mostafa ${ }^{1}$, M. Murai ${ }^{1}$, R. Nishimura ${ }^{1}$, O. Fujita ${ }^{2}$ and Y. Nihei ${ }^{3}$}

${ }^{1}$ Dept. of Environment and System Sciences, Yokohama National University, Yokohama 240-0066, Japan, Email: nmostafa79@gmail.com

${ }^{2}$ Imabari Shipbuilding Co. Ltd., Marugame city 799-2195, Japan

${ }^{3}$ Dept. of Aerospace and Marine System Engineering, Osaka Prefecture University, Osaka 599-8531, Japan

\begin{abstract}
:
Recently, a number of research groups have paid much attention to the study of Floating Offshore Wind Turbines (FOWTs). Similar to other offshore structures, the FOWTs are subjected to irregular waves and wind loads which cause a dynamic response in the structures. Under marine environmental conditions, they face many forces which prevent them from floating in the upright condition; they incline as a result of the winds, strong currents, typhoons, cyclones, storms etc. The motion of the FOWT might be changed by a change in gyroscopic effect which depends on the angular velocity and moment of inertia of the blade. Therefore, to investigate the effect of the gyro moment on the motion of the FOWT, two types of experiment were carried out in a water tank using a 1/360 scale model of a prototype FOWT. Firstly, the interaction between the rotary motion of the wind turbine blade and the dynamic motion of the SPAR-type FOWT was studied at small angles of inclination in regular waves. Secondly, the interaction between the change of rotational speed as well as moment of inertia of the blade and the motion of the FOWT was studied. In this paper, numerical calculations have been carried out using potential theory based on the $3 D$ panel method. Finally, the experimental results are compared with the results of numerical simulation and findings are discussed.
\end{abstract}

Keywords: Floating offshore wind turbine, SPAR, moment of inertia, gyro moment, inclination etc.

\section{Introduction}

Nowadays, the energy problem is one of the fundamental issues facing the world. The development of renewable energy resources will help to reduce the effects of global warming. As global warming and exhaustion of fossil fuels become a serious issue, the development of alternative energy sources such as hydro energy, solar energy and wind energy has become imperative. It is widely known that the vast marine space is one of the main sources of renewable energy. The offshore expansion of wind turbines is becoming increasingly common, especially in Europe. In future, it is expected that floating offshore wind turbines (FOWTs) will become a possible option. According to the Report of Ministry of Economy, Trade and Industry and European Wind Energy, Japan and the European Union aims to produce $20.0 \%$ of their electricity from renewable energy. Therefore, many groups researching renewable energy are looking into the development of cost-effective FOWTs. Currently, there are a number of offshore wind turbines with floating foundation concepts in various stages of development. They are divided into four main categories: Spars-buoy type (SPAR-type), Tension leg platform type, Semi-submersible type and Barge-type. In general, the SPAR-type has a better heave performance than the Semi-submersible type due to its deep draft and reduced vertical wave exciting forces, as shown by Roddier et al. (2010). Therefore, the authors have selected the SPAR-type FOWT as the basis of their research because of its simpler shape and expected cost-effectiveness. Figure 1 shows a SPAR-type FOWT concept which was proposed by StatoilHydro's Hywind project as a test case in Norway.

Up to the present time, several types of FOWT have been proposed such as, by Musial et al. (2004) and Butterfield et al. (2005). In particular, StatoilHydro (2009) has installed a full scale SPAR-type FOWT, the Hywind project, offshore in Norway. Nielsen et al. (2006) and Skaare et al. (2007) examined the dynamic response of this SPAR-type FOWT under the action of winds and waves by experiment and numerical 
N.Mostafa, M. Murai, R.Nishimura, O. Fujita and Y. Nihei/Journal of Naval Architecture and Marine Engineering 9(2012) 67-79 simulation. Several concepts of FOWT have been proposed and are under research in Japan by Fukumoto et al. (2006), Suzuki et al. (2007) and Utsunomiya et al. (2010).

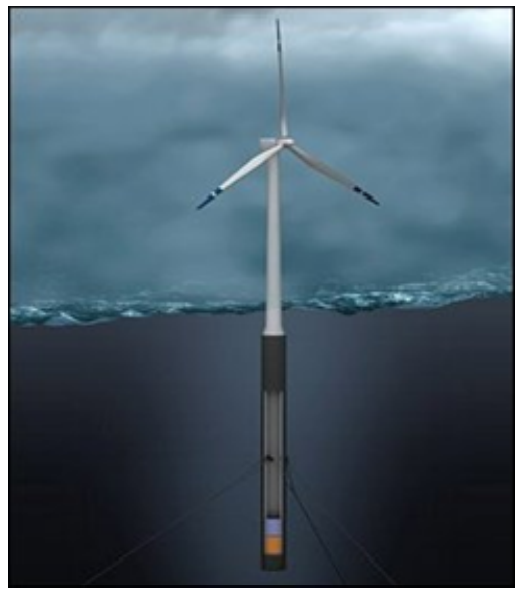

Fig. 1: Floating foundation concept for offshore wind turbine (Hywind project, 2008)

Many problems have to be overcome before the FOWT can be considered suitable for practical use. Generally, the motions induced by waves and currents are a serious problem. The tower of a ground type wind turbine is fixed; the change in the rotor surface on revolution of the blades is extremely small. In contrast, in the case of the floating type wind turbine, the whole floating structure is affected by sea waves. Therefore, the rotor surface of the wind turbine always vibrates during revolution. It is well known that a rigid body having a rotating wheel has a moment of gyro effect from the change in the direction of the wheel. In the FOWT, this effect is clearly present.

The aim of the present study is to investigate numerically and experimentally the relationship between the motion of the FOWT and the change in gyroscopic effect induced by the change in inertia of the blade of the FOWT. Also the dynamic motion of the SPAR-type FOWT is investigated having a small steady inclination angle in waves. Numerical calculations have been carried out using potential theory based on the 3D panel method.

\section{Formulation}

\subsection{Equation of motion}

Let $(x, y, z)$ be the right-handed Cartesian coordinate system with the $x y$ plane on the mean free surface and $z$ the axis directed vertically upwards through the center of the FOWT as shown in Fig. 2. The motion of the FOWT in waves can be represented by equation of motion with six degrees freedom. The six degrees of freedom are composed of three translational motions and three angular motions. The translational displacements in the $x, y, z$ directions are $\xi_{1}, \xi_{2}, \xi_{3}$, respectively, where $\xi_{1}$ is the surge, $\xi_{2}$ is the sway, and $\xi_{3}$ is the heave motion. Furthermore, the angular displacements of the motions about the $x, y, z$ axes are $\xi_{4}, \xi_{5}, \xi_{6}$, respectively, where $\xi_{4}$ is the roll, $\xi_{5}$ is the pitch and $\xi_{6}$ is the yaw angle.

The dynamic equation of motion in the frequency domain of a FOWT in waves can be written as follows:

$$
\{(\mathbf{M}+\mathbf{m}) \ddot{\xi}+\mathbf{N} \dot{\xi}+\mathbf{C} \xi\}=\mathbf{F}
$$

where $\mathbf{M}$ is the $6 \times 6$ mass matrix, $\mathbf{m}$ is the $6 \times 6$ added mass matrix, and $\mathbf{N}$ is the $6 \times 6$ damping matrix of the FOWT. The added mass and the damping are related to the hydrodynamic radiation force. The former one is proportional to the acceleration of the motion and the latter is proportional to the velocity of the motion. $\mathbf{C}$ is the $6 \times 6$ restoring force matrix, $\xi$ is the motion response vector with 6 degrees of freedom. $\mathbf{F}$ is the vector of wave exciting forces with 6 degrees of freedom. 


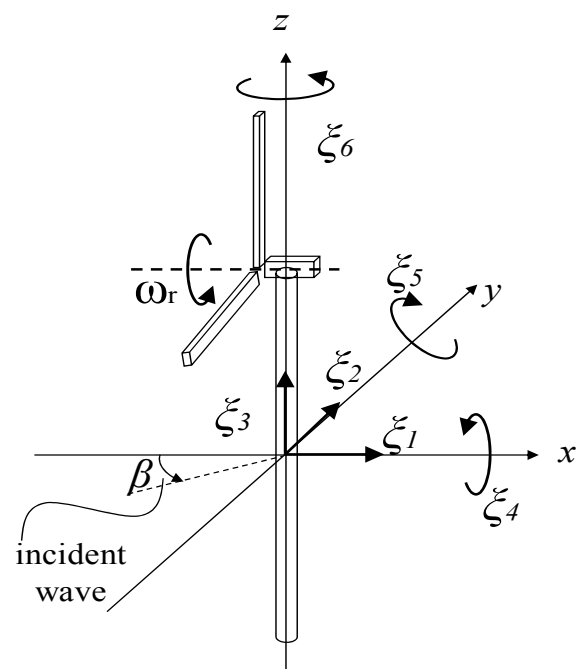

Fig. 2: Coordinate system of the FOWT

When the rotor of the FOWT is turned, there is an induced gyroscopic force which has an influence on the motion of the FOWT. Therefore, the equation of gyro moment is added to the dynamic equation of motion of the FOWT. The additional coordinate system proposed by Murai et al. (2010) to expresses the rotational motion of blades is shown in Fig. 3. The center of the blades which rotate with angular velocity $\omega_{r}(=$ const $)$ is at Or. The rotary axis of the blades is defined as the $x$ axis as shown in Fig. 2.

Let $\left(x_{r}, y_{r}, z_{r}\right)$ be the right-handed Cartesian coordinate system of the rotating blades with $x_{r}$ and $y_{r}$ in the direction of the horizontal plane perpendicular to each other and the $z_{r}$ axis directed vertically upwards (see Fig. 3). Assume that the vibrational angle of the blades around the $x_{r}$ axis is $\xi_{r 4}$, around the $y_{r}$ axis is $\xi_{r 5}$ and around the $z_{r}$ axis is $\xi_{r 6}$. The angular velocity of these angles depends on the angular velocity $\omega$ of the periodic external force. These angles are defined as follows:

$$
\begin{aligned}
& \xi_{r 4}=\xi_{r 4}^{0} e^{-i \omega t} \\
& \xi_{r 5}=\xi_{r 5}^{0} e^{-i \omega t} \\
& \xi_{r 6}=\xi_{r 6}^{0} e^{-i \omega t}
\end{aligned}
$$

Here, $\xi_{r 4}^{0}, \xi_{r 5}^{0}, \xi_{r 6}^{0}$ on the right side of Eq. (2) show the amplitudes of the rotational angle of the motion. Therefore, the angular velocities $\omega_{r 4}, \omega_{r 5}, \omega_{r 6}$ around the respective axis can be written as

$$
\begin{aligned}
& \omega_{r 4}=\omega_{r}-i \omega \xi_{r 4}^{0} e^{-i \omega t} \\
& \omega_{r 5}=-i \omega \xi_{r 5}^{0} e^{-i \omega t} \\
& \omega_{r 6}=-i \omega \xi_{r 6}^{0} e^{-i \omega t}
\end{aligned}
$$

Differentiating this equation with respect to time, the following equation is obtained.

$$
\begin{aligned}
& \dot{\omega}_{r 4}=-\omega^{2} \xi_{r 4}^{0} e^{-i \omega t} \\
& \dot{\omega}_{r 5}=-\omega^{2} \xi_{r 5}^{0} e^{-i \omega t} \\
& \dot{\omega}_{r 6}=-\omega^{2} \xi_{r 6}^{0} e^{-i \omega t}
\end{aligned}
$$

In order to obtain the moments for each axis, these equations are substituted into Euler's equations of motion. Euler's equations are represented by using the moments $M_{r 4}, M_{r 5}$ and $M_{r 6}$, and are shown as,

$$
\begin{aligned}
& M_{r 4}=I_{r 1} \dot{\omega}_{r 4}-\left(I_{r 2}-I_{r 3}\right) \omega_{r 5} \omega_{r 6} \\
& M_{r 5}=I_{r 2} \dot{\omega}_{r 5}-\left(I_{r 3}-I_{r 1}\right) \omega_{r 6} \omega_{r 4} \\
& M_{r 6}=I_{r 3} \dot{\omega}_{r 6}-\left(I_{r 1}-I_{r 2}\right) \omega_{r 4} \omega_{r 5}
\end{aligned}
$$




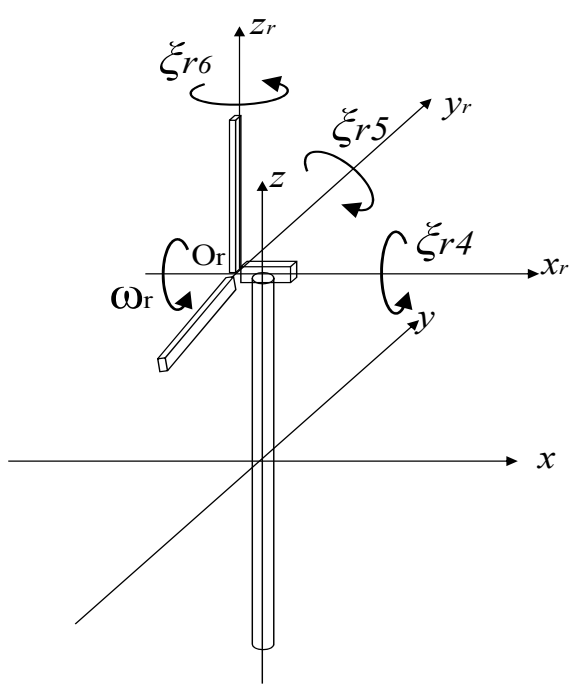

Fig. 3: Coordinate system for the rotational motion of the blades

where $I_{r 1}, I_{r 2}$ and $I_{r 3}$ are the moments of inertia of the blades around the respective axis. The following equation can be obtained after substituting Eqs. (3) and (4) into Eq. (5).

$$
\begin{aligned}
& M_{r 4}=-I_{r 1} \omega^{2} \xi_{r 4}^{0} e^{-i \omega t}+\left(I_{r 2}-I_{r 3}\right) \omega^{2} \xi_{r 5}^{0} \xi_{r 6}^{0} e^{-2 i \omega t} \\
& M_{r 5}=-I_{r 2} \omega^{2} \xi_{r 5}^{0} e^{-i \omega t}+\left(I_{r 3}-I_{r 1}\right) i \omega \xi_{r 6}^{0} e^{-i \omega t}\left(\omega_{r}-i \omega \xi_{r 4}^{0} e^{-i \omega t}\right) \\
& M_{r 6}=-I_{r 3} \omega^{2} \xi_{r 6}^{0} e^{-i \omega t}+\left(I_{r 1}-I_{r 2}\right) i \omega \xi_{r 5}^{0} e^{-i \omega t}\left(\omega_{r}-i \omega \xi_{r 4}^{0} e^{-i \omega t}\right)
\end{aligned}
$$

By considering the vibrational amplitude of Eq. (6) to be small, the product of vibrational amplitude can be ignored, and Eq. (6) can be written as Eq. (7):

$$
\begin{aligned}
& M_{r 4}=-I_{r 1} \omega^{2} \xi_{r 4}^{0} e^{-i \omega t} \\
& M_{r 5}=-I_{r 2} \omega^{2} \xi_{r 5}^{0} e^{-i \omega t}+\left(I_{r 3}-I_{r 1}\right) i \omega_{r} \omega \xi_{r 6}^{0} e^{-i \omega t} \\
& M_{r 6}=-I_{r 3} \omega^{2} \xi_{r 6}^{0} e^{-i \omega t}+\left(I_{r 1}-I_{r 2}\right) i \omega_{r 1} \omega \xi_{r 5}^{0} e^{-i \omega t}
\end{aligned}
$$

The 2nd term on the right-hand side of Eq. (7) for $M_{r 5}$ and $M_{r 6}$, represents the gyro moment of rotation of the blades. Therefore, the gyro moment is shown in the following dynamic equation of motion.

$$
\left\{-\omega^{2}(\mathbf{M}+\mathbf{m})-i \omega\left(\mathbf{N}-\mathbf{M}_{\text {gyro }}\right)+\mathbf{C}\right\} \xi=\mathbf{F}
$$

where $\mathbf{M}_{\text {gyro }}$ is written as follows:

$$
\mathbf{M}_{\text {gyro }}=\left[\begin{array}{cccccc}
0 & 0 & 0 & 0 & 0 & 0 \\
0 & 0 & 0 & 0 & 0 & 0 \\
0 & 0 & 0 & 0 & 0 & 0 \\
0 & 0 & 0 & 0 & 0 & 0 \\
0 & 0 & 0 & 0 & 0 & \left(I_{r 3}-I_{r 1}\right) \omega_{r} \\
0 & 0 & 0 & 0 & \left(I_{r 1}-I_{r 2}\right) \omega_{r} & 0
\end{array}\right]
$$

Finally, when the inverse matrix of the matrix of the left-hand side of Eq. (8) is multiplied by both sides of Eq. (8), the dynamic equation is obtained as follows:

$$
\xi=\left\{-\omega^{2}(\mathbf{M}+\mathbf{m})-i \omega\left(\mathbf{N}-\mathbf{M}_{\text {gyro }}\right)+\mathbf{C}\right\}^{-1} \mathbf{F}
$$

\subsection{Coordinate transformation of moment of inertia}

In this study, it is considered that the moment of inertia of the model depends on coordinate transformation. Therefore, it is necessary to determine the moment of inertia around the center of the FOWT at the angle of inclination. Assume the values of the moment of inertia $I_{l x}, I_{2 y}$ and $I_{3 z}$ with respect to the $\mathrm{x}, \mathrm{y}$, and $\mathrm{z}$ axes to be known. Determine the values of the moment of inertia $I_{1 u}, I_{2 v}$ and $I_{3 w}$ with respect to the $u, v$, and $w$ axes, which are inclined at an angle $\theta$ to the $x$ and $\mathrm{z}$ axes, as shown in Fig. 4. 


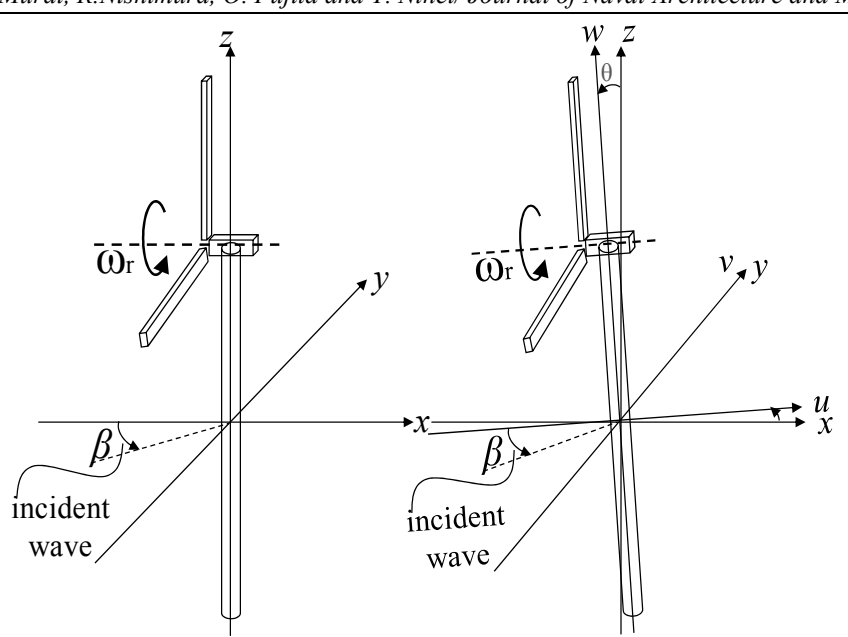

Fig. 4: Moment of inertia with respect to the inclined axes

Therefore, the moment of inertia of the FOWT with respect to the angle $\theta$ is as follows:

$$
\begin{aligned}
& I_{1 u}=\frac{I_{1 x}+I_{3 z}}{2}+\frac{I_{1 x}-I_{3 z}}{2} \cos 2 \theta \\
& I_{2 v}=I_{2 y} \\
& I_{3 w}=\frac{I_{1 x}+I_{3 z}}{2}-\frac{I_{1 x}-I_{3 z}}{2} \cos 2 \theta
\end{aligned}
$$

From Eq. (1), the mass matrix of the FOWT in the direction of the upright condition, which has $x z$ plane symmetry, can be written as:

$$
\mathbf{M}=\left[\begin{array}{cccccc}
m & 0 & 0 & 0 & m z_{G} & 0 \\
0 & m & 0 & -m z_{G} & 0 & 0 \\
0 & 0 & m & 0 & 0 & 0 \\
0 & -m z_{G} & 0 & I_{1 x} & 0 & 0 \\
m z_{G} & 0 & 0 & 0 & I_{2 y} & 0 \\
0 & 0 & 0 & 0 & 0 & I_{3 z}
\end{array}\right]
$$

where $m$ is the mass of the FOWT, $z_{G}$ is the vertical coordinate of the center of gravity in the body fixed coordinate system and $I_{1 x}, I_{2 y}$, and $I_{3 z}$ are the moments of inertia of the FOWT with respect to the $x, y$, and $z$ axes, respectively.

Considering Eqs. (11) (13), the generalized mass matrix of the FOWT at the angle of inclination can be written as:

$$
\mathbf{M}=\left[\begin{array}{cccccc}
m & 0 & 0 & 0 & m z_{G} & 0 \\
0 & m & 0 & -m z_{G} & 0 & 0 \\
0 & 0 & m & 0 & 0 & 0 \\
0 & -m z_{G} & 0 & I_{1 u} & 0 & 0 \\
m z_{G} & 0 & 0 & 0 & I_{2 v} & 0 \\
0 & 0 & 0 & 0 & 0 & I_{3 w}
\end{array}\right]
$$

Now substituting Eq. (15) into Eq. (1), the dynamic equation of motion of the FOWT is obtained at the angle of inclination. 
N.Mostafa, M. Murai, R.Nishimura, O. Fujita and Y. Nihei/Journal of Naval Architecture and Marine Engineering 9(2012) 67-79

\section{Model Experiment}

\subsection{Experimental set-up}

In order to clarify the effects of change of gyro moment and the motion of the FOWT at the angle of inclination, simple experiments were carried out in a water tank at Yokohama National University. The dimensions of the water tank were $16 \mathrm{~m}$ long, $1 \mathrm{~m}$ wide and $2 \mathrm{~m}$ deep. A plunger type wave maker was located at one end of the tank and a wave absorption beach at the other end. Regular waves were generated by the wave maker in water $1.5 \mathrm{~m}$ deep. As shown in Fig. 5, the assumed FOWT model was proposed by Suzuki et al. (2005). The experimental model of the SPAR-type FOWT is shown as a photo and sketch in Fig. 6. The parameters of the FOWT model are listed in Table 1.

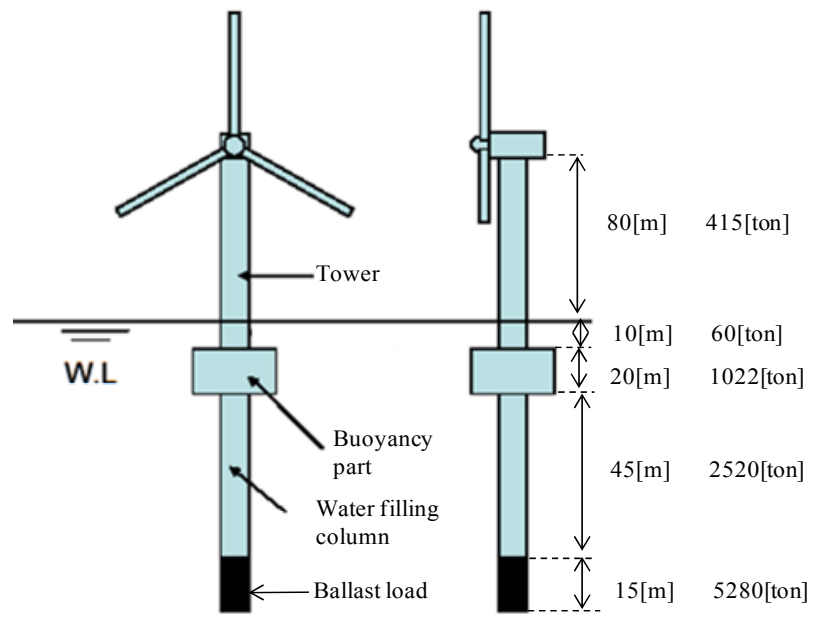

Fig. 5: Rough sketch of concepts of the SPAR-type FOWT

Table 1: Model parameters

\begin{tabular}{|l|c|c|}
\hline \multicolumn{1}{|c|}{ Specimen } & Experimental model & Actual model \\
\hline Tower height & $226.0(\mathrm{~mm})$ & $80.0(\mathrm{~m})$ \\
\hline Diameter of buoyancy part & $70.0(\mathrm{~mm})$ & $20.0(\mathrm{~m})$ \\
\hline Height of buoyancy part & $54.0(\mathrm{~mm})$ & $20.0(\mathrm{~m})$ \\
\hline Water plane area & $315.0 \sim 318.0\left(\mathrm{~mm}^{2}\right)$ & $201.1\left(\mathrm{~m}^{2}\right)$ \\
\hline Draft & $277.0(\mathrm{~mm})$ & $95.0(\mathrm{~m})$ \\
\hline Total mass & $245.6(\mathrm{~g})$ & $10047.0($ ton$)$ \\
\hline Height of center of gravity from bottom & $153.0(\mathrm{~mm})$ & $35.0(\mathrm{~m})$ \\
\hline Metacentric height $(\mathrm{GM})^{4}$ & $42.3 \sim 45.0(\mathrm{~mm})$ & $24.0(\mathrm{~m})$ \\
\hline Spring constant for mooring & $7.7(\mathrm{~N} / \mathrm{m})$ & - \\
\hline
\end{tabular}

${ }^{4}$ Here, "GM" is the distance from the center of gravity and the metacenter of a FOWT

A flat blade with two small "weights A" was used as the rotor in the experimental model, as shown in Fig. 7. No attempts were made to create the wind force. A motor was placed at the top of the tower to create the gyroscopic effect. This well-known mechanical force arises when a rotor rotating around a certain axis undergoes a rotation around a different axis. The motor model was PNN13RE09HD from Minabea Motor Manufacturing Corporation. The tower part and the buoyancy part were made of balsa and the column which was under water was made of polycarbonate pipe. Then ballast was used inside the column of the end part. 

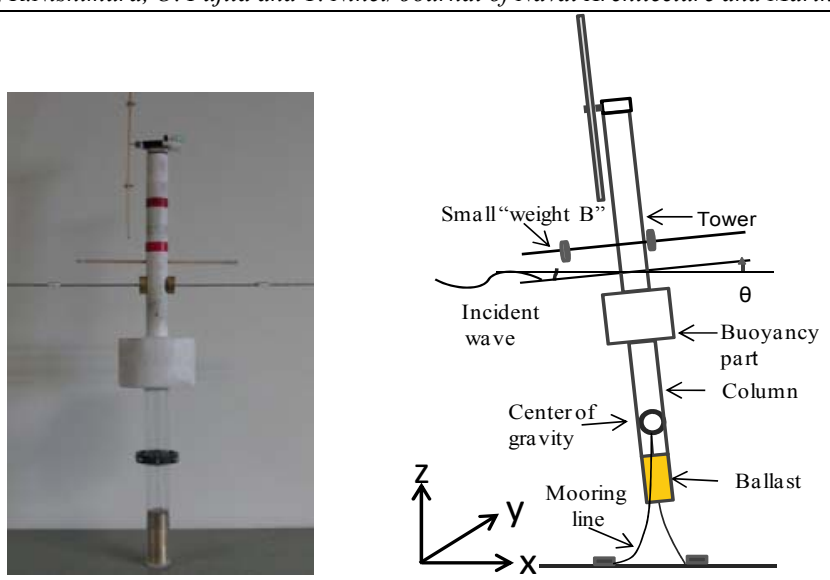

Fig. 6: Photo (left) and sketch (right) of the experimental model

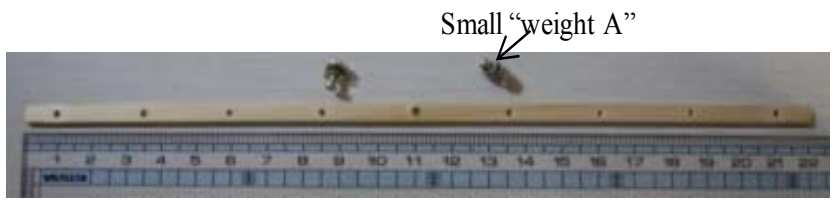

Fig. 7: Rotating flat blade with weights

Table 2: Dimensions of flat blade

\begin{tabular}{|c|c|}
\hline Dimension & Units \\
\hline Length & $220.0(\mathrm{~mm})$ \\
\hline Width & $5.0(\mathrm{~mm})$ \\
\hline Thickness & $2.0(\mathrm{~mm})$ \\
\hline Mass & $0.9(\mathrm{~g})$ \\
\hline Total mass (including small "weights A") & $2.0(\mathrm{~g})$ \\
\hline
\end{tabular}

Table 3: Effect of change of position of "weight A" on the flat blade

\begin{tabular}{|c|c|c|c|c|c|c|}
\hline $\begin{array}{c}\text { Distance from } \\
\text { the center of } \\
\text { blade to the } \\
\text { "weight A", d } \\
(\mathrm{mm})\end{array}$ & $\begin{array}{c}\text { Angular } \\
\text { velocity } \\
\omega_{r} \\
(\mathrm{rad} / \mathrm{s})\end{array}$ & $\begin{array}{c}\text { Moment of } \\
\text { inertia } \\
I_{r 1}\left(\mathrm{~kg} \cdot \mathrm{m}^{2}\right)\end{array}$ & $\begin{array}{c}\text { Moment of } \\
\text { inertia } \\
I_{r 2}\left(\mathrm{~kg} \cdot \mathrm{m}^{2}\right)\end{array}$ & $\begin{array}{c}\text { Moment of } \\
\text { inertia } \\
I_{r 3}\left(\mathrm{~kg} \cdot \mathrm{m}^{2}\right)\end{array}$ & $\left(I_{r 1}-I_{r 2}\right) \omega_{r}$ & $\left(I_{r 3}-I_{r 1}\right) \omega_{r}$ \\
\hline 25.0 & 42.0 & $4.43 \times 10^{-7}$ & $2.38 \times 10^{-7}$ & $2.38 \times 10^{-7}$ & $8.64 \times 10^{-6}$ & $-8.64 \times 10^{-6}$ \\
\hline 50.0 & 38.5 & $5.39 \times 10^{-7}$ & $3.33 \times 10^{-7}$ & $3.33 \times 10^{-7}$ & $7.92 \times 10^{-6}$ & $-7.92 \times 10^{-6}$ \\
\hline 75.0 & 35.0 & $6.98 \times 10^{-7}$ & $4.93 \times 10^{-7}$ & $4.93 \times 10^{-7}$ & $7.19 \times 10^{-6}$ & $-7.19 \times 10^{-6}$ \\
\hline 100.0 & 32.0 & $9.21 \times 10^{-7}$ & $7.16 \times 10^{-7}$ & $7.16 \times 10^{-7}$ & $6.58 \times 10^{-6}$ & $-6.58 \times 10^{-6}$ \\
\hline
\end{tabular}

First, the variation of angular velocity of the rotating flat blade was measured when the small "weights A" were positioned at different locations on the flat blade. The holes in the flat blade were located every $25.0 \mathrm{~mm}$ from the center of rotation. The dimensions of the flat blade are listed in Table 2. To calculate the angular velocity of the rotation, a CCD Laser Displacement Sensor was used. The change of gyroscopic effect depends on the change of angular velocity and moment of inertia of the rotating flat blade. In order to calculate the change of angular velocity as well as the change of moment of inertia of the flat blade, the attached "weights A" were varied from $25.0 \mathrm{~mm}$ to $100.0 \mathrm{~mm}$ from the center of the flat blade and results are listed in Table 3 . From Table 3 , it is concluded that the angular velocity of the blade decreases with increasing the distance from the center of the flat blade. But the moment of inertia increases with increasing the distance from the center of the flat blade. 
N.Mostafa, M. Murai, R.Nishimura, O. Fujita and Y. Nihei/ Journal of Naval Architecture and Marine Engineering 9(2012) 67-79 The measured change of gyroscopic effect is higher at $\mathrm{d}=25.0[\mathrm{~mm}]$ and decreases with increasing the distance. For each condition, a water tank experiment was carried out.

\subsection{Experimental method}

A schematic diagram of the water tank with the experimental model is depicted in Fig. 8. In order to prevent the experimental model from drifting, it was moored by two spring mooring lines. The mooring lines were connected to the center of gravity of the model in the $y$ direction. The mooring stiffness was measured by experiment and the measured stiffness is $7.7 \mathrm{~N} / \mathrm{m}$. The effect of mooring stiffness cannot be ignored. So, the value of mooring stiffness is added directly to the restoring force coefficient in numerical computation. The experiments were carried out with wave periods of $0.5,0.6,0.7,0.8,0.9,1.0,1.1$, and $1.2 \mathrm{~s}$ for $0^{\circ}$ wave heading (head waves). In each wave period, a video of the surge, heave, pitch and yaw motion of the model was recorded with two digital cameras for 4 second of real time and analyzed an image of the animation using the color tracking method.

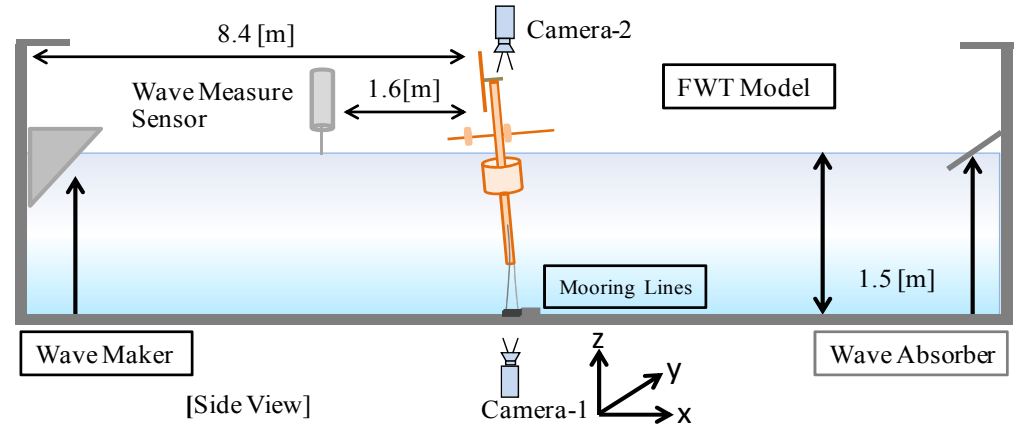

Fig. 8: Experimental set-up in water tank
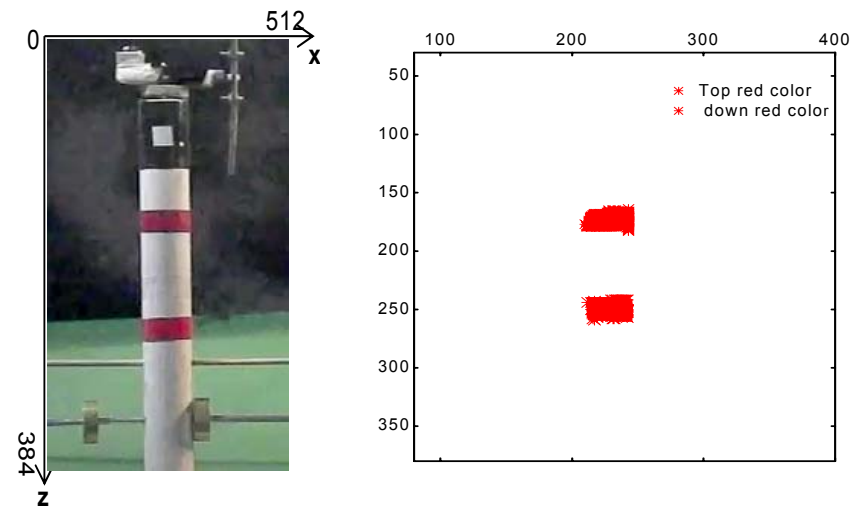

(a) Picture from Camera-1 and analyzed position of tapes
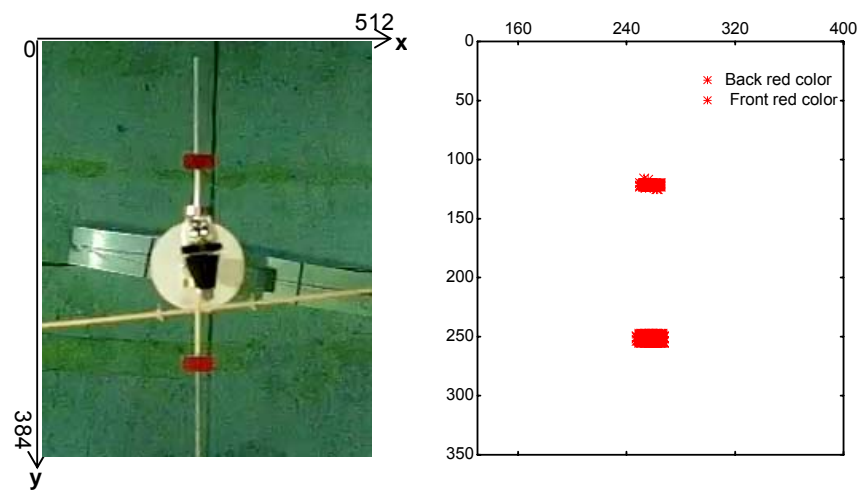

(b) Picture from Camera-2 and analyzed position of tapes

Fig. 9: Sample result showing photo of the model and the data; (a) Camera-1 and (b) Camera-2 
N.Mostafa, M. Murai, R.Nishimura, O. Fujita and Y. Nihei/ Journal of Naval Architecture and Marine Engineering 9(2012) 67-79

Two red marker tapes were placed on the vertical tower part of the model (see Fig. 9a) and also two red tapes were placed on the horizontal light bar which was connected to the tower of the model (see Fig.9b). Using camera-1, pictures of the motion of the red marks were taken in the $x z$ plane (see Fig.9a) and analyzed their surge, heave and pitch motion. On the other hand, camera- 2 was used to take pictures of the motion of the red marks in the $x y$ plane (see Fig. 9b) and analyzed their yaw motion. Using the data from both cameras', the response of the motion was obtained by Fourier analysis.

A set method was followed for video analysis. The animation was separated which had been recorded with digital cameras (300 frames per second) into still images. The position of each tape was read from the still image and read the time trajectory of the tape. This time trajectory of the tape shows the time series response of the motion of the model. A sample result showing a photo of the model in the waves and the analysis data is shown in Fig. 9.

\section{Results and Discussion}

\subsection{The influence of gyro moment}

Using the time series response of each motion, graphs of the frequency response of the surge, heave, pitch, and yaw motion are obtained and compare those results to the results of numerical computation. The response amplitude operator (RAO) of the motion responses of surge, and heave is defined by the ratio of motion amplitude and incident wave amplitude $\left(\xi_{a}\right)$. On the other hand, the RAO of pitch and yaw is defined by the ratio of motion amplitude and wave amplitude times wave number $(k)$. The RAO is a function of normalized frequency, $2 r \omega^{2} / g$ where $r$ is the radius of the buoyancy part of the experimental model, $\omega$ is the wave frequency and $g$ is the gravity acceleration. In the figures, symbols show the experimental results and lines represent numerical calculations.

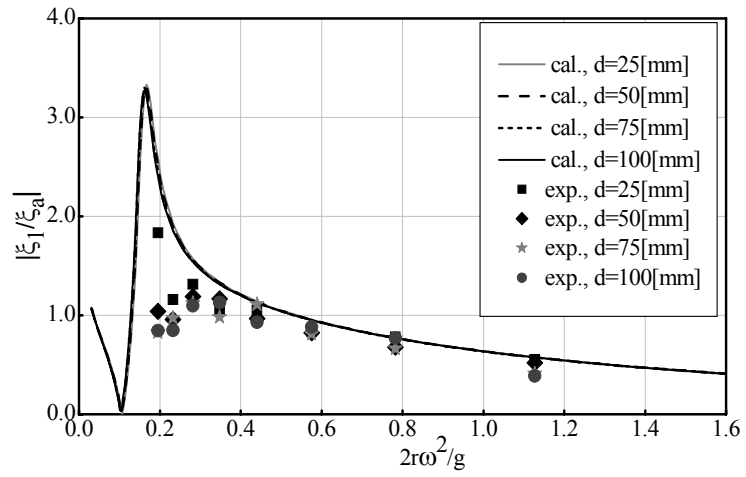

(a) Surge

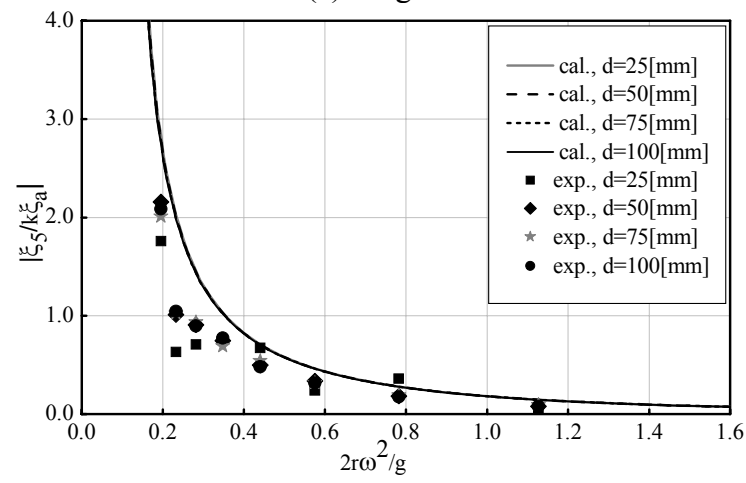

(c) Pitch

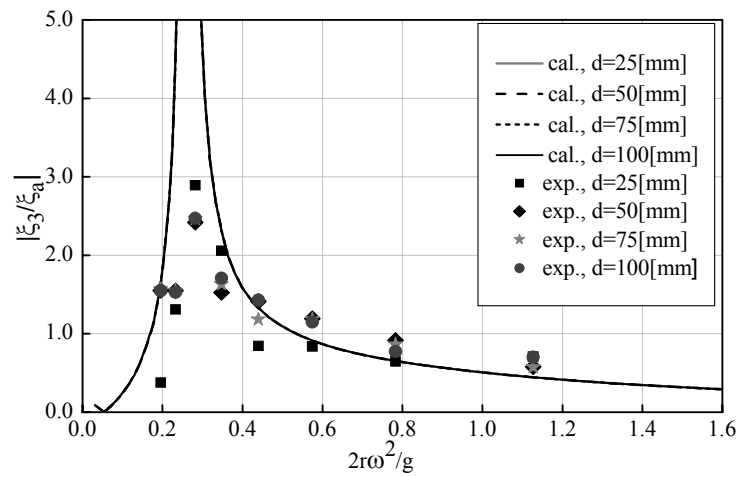

(b) Heave

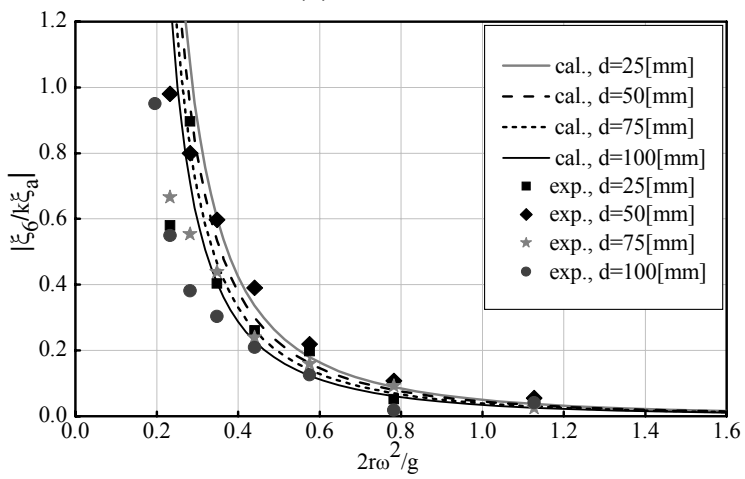

(d) Yaw

Fig. 10: RAO of (a) Surge; (b) Heave (c) Pitch; (d) Yaw motion at 0 degree angle of inclination

Figure 10 illustrates the results of change of gyroscopic effect with the change of location of small "weights A" on the flat blade. The location of small "weights A" from the center of the blade was taken as $d=25.0,50.0$, 75.0 , and $100.0 \mathrm{~mm}$. The change of gyroscopic effect does not affect the surge, heave, and pitch motion, but its 
N.Mostafa, M. Murai, R.Nishimura, O. Fujita and Y. Nihei/ Journal of Naval Architecture and Marine Engineering 9(2012) 67-79 effects appear on yaw motion. A resonant period of heave motion is seen near the normalized frequency, $2 r \omega^{2} / g=0.28$. At frequencies above 0.28 , the RAOs of surge, heave, pitch and yaw show a comparable response with the change of gyroscopic effect. But discrepancies are observed in the experimental result for normalized frequencies lower than 0.28 . It can be considered that the main reason for such discrepancies is that the small scale model is not effective at lower frequencies. Hence, the responses between the normalized frequencies 0.28 to 1.13 are discussed. Peak response is observed in surge and heave motion in numerical computation. This response may be due to the fact that the damping effect is not considered in the numerical computation. From Eq. 9, it is seen that the yaw motion of the FOWT is affected by the gyro moment, which is clearly seen in numerical computations and experimental results at normalized frequencies above 0.28 (see Fig. 10). Figure 11 shows the results of RAO of yaw motion at the normalized frequencies 0.44 and 0.57 with the change of gyro moment of the rotating flat blade. This linear relationship demonstrates that the yaw response is increased with increase in the change of gyro effect (see Table 3). As the change of gyro moment implies a lower response, the higher moment of inertia of the rotating blade plays an essential role in yaw response. Therefore, the change of gyro moment (discussed in Section 2) has an effect on the numerical and experimental results for yaw motion of the FOWT (see Fig. 11).

\subsection{Response of a FOWT with change of angle of inclination}

When a horizontal force is applied to the FOWT, it causes inclination. The response of motion of a FOWT is examined with the change of angle of inclination. A number of variable angles $\theta=0.0,2.0,4.0,6.0$, and 8.0 degrees were measured by analyzing the recorded video of the FOWT experimental model. To create these angles, "weight B" was shifted away from the tower of the model in the negative direction of the $x$ axis (see Fig. 6 ). The location of the small "weights $A$ " on the blade was kept constant at $25.0 \mathrm{~mm}$ away from the center of rotation of the flat blade throughout the experiments.

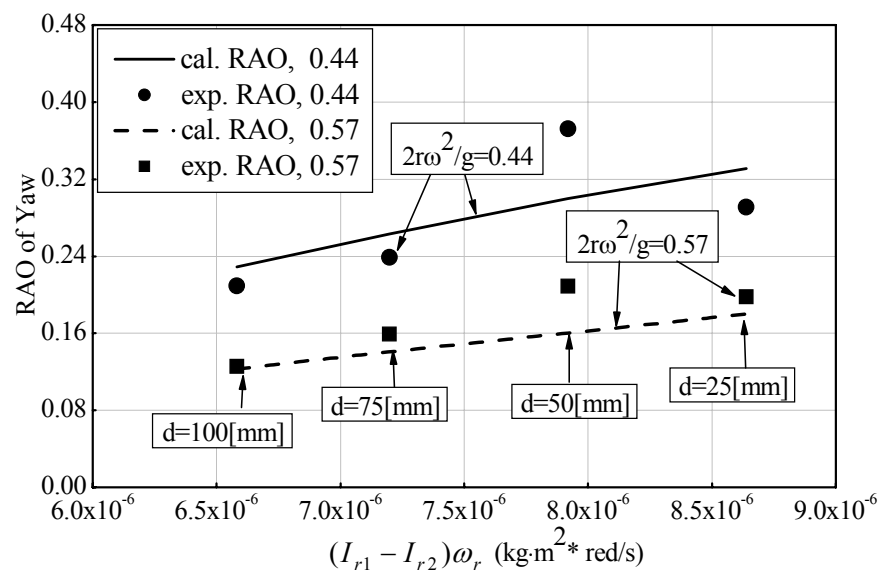

Fig. 11: RAO of yaw with the variation of the moment of inertia of the blades for normalized frequencies, $2 r \omega^{2} / g=0.44$ and 0.57

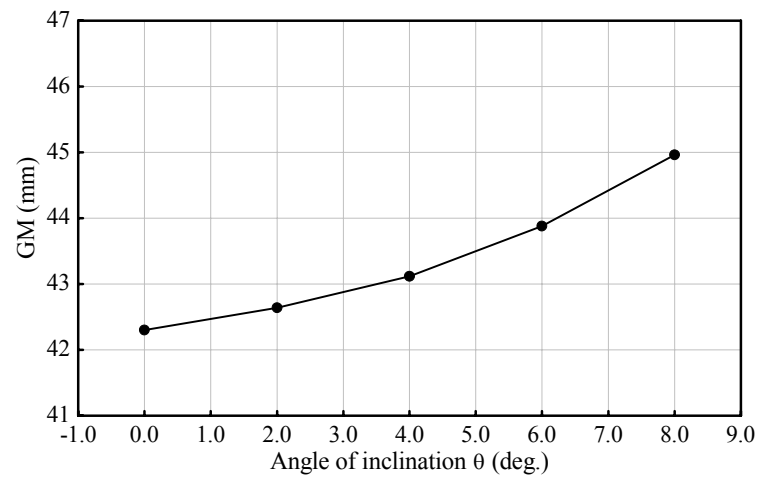

Fig.12: Relationship between the angle of inclination and the metacentric height of the FOWT 
N.Mostafa, M. Murai, R.Nishimura, O. Fujita and Y. Nihei/ Journal of Naval Architecture and Marine Engineering 9(2012) 67-79 A relationship between the angle of inclination and the metacentric height (GM) of the FOWT is illustrated in Fig. 12. When the "weight B" is shifted (see Fig. 6), the center of buoyancy shifts as the angle of inclination increases and the water plane area increases (see Table 1). As shown in this figure, the metacentric height increases with increasing angle of inclination.

The moments of inertia of the FOWT at the angle of inclination are calculated with Eqs. 11 13 and the values are listed in Table 4. As can be seen, the moment of inertia around the $w$ axis increases with increasing the angle of inclination but the moment of inertia around the $u$ axis decreases with increasing angle of inclination. The moment of inertia around the $v$ axis is constant with the change of angle of inclination (see Eq. (12)).

Table 4: Moment of inertia at the angle of inclination

\begin{tabular}{|c|c|c|c|}
\hline $\begin{array}{c}\text { Angle of inclination, } \\
\theta \text { (deg.) }\end{array}$ & $\begin{array}{c}\text { Moment of inertia, } \\
I_{1 u}\left(\mathrm{~kg} \cdot \mathrm{m}^{2}\right)\end{array}$ & $\begin{array}{c}\text { Moment of inertia, } \\
I_{2 v}\left(\mathrm{~kg} \cdot \mathrm{m}^{2}\right)\end{array}$ & $\begin{array}{c}\text { Moment of inertia, } \\
I_{3 w}\left(\mathrm{~kg} \cdot \mathrm{m}^{2}\right)\end{array}$ \\
\hline 0.0 & $6.26 \times 10^{-4}$ & $6.26 \times 10^{-4}$ & $3.53 \times 10^{-6}$ \\
\hline 2.0 & $6.25 \times 10^{-4}$ & $6.26 \times 10^{-4}$ & $5.13 \times 10^{-6}$ \\
\hline 4.0 & $6.23 \times 10^{-4}$ & $6.26 \times 10^{-4}$ & $7.58 \times 10^{-6}$ \\
\hline 6.0 & $6.19 \times 10^{-4}$ & $6.26 \times 10^{-4}$ & $1.11 \times 10^{-5}$ \\
\hline 8.0 & $6.14 \times 10^{-4}$ & $6.26 \times 10^{-4}$ & $1.62 \times 10^{-5}$ \\
\hline
\end{tabular}

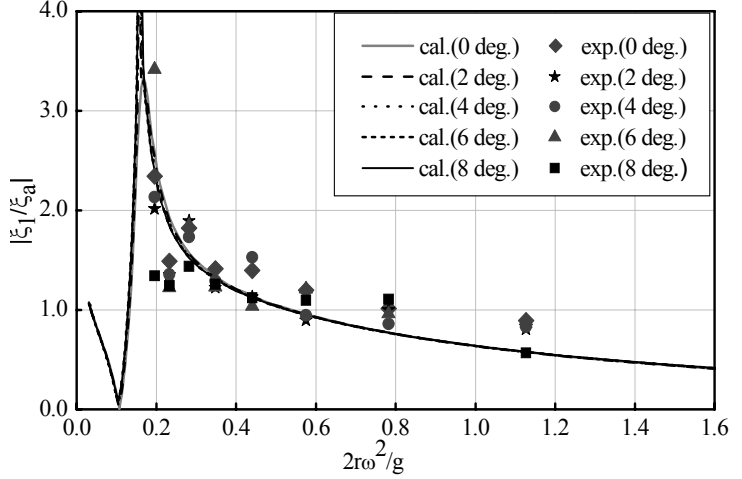

(a) Surge

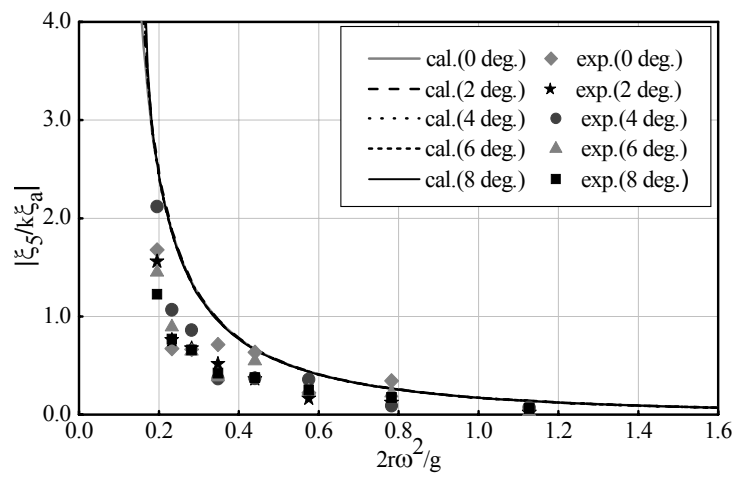

(c) Pitch

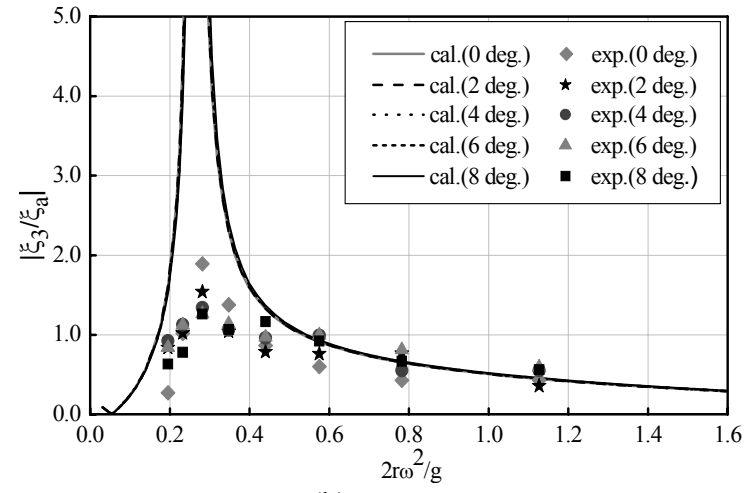

(b) Heave

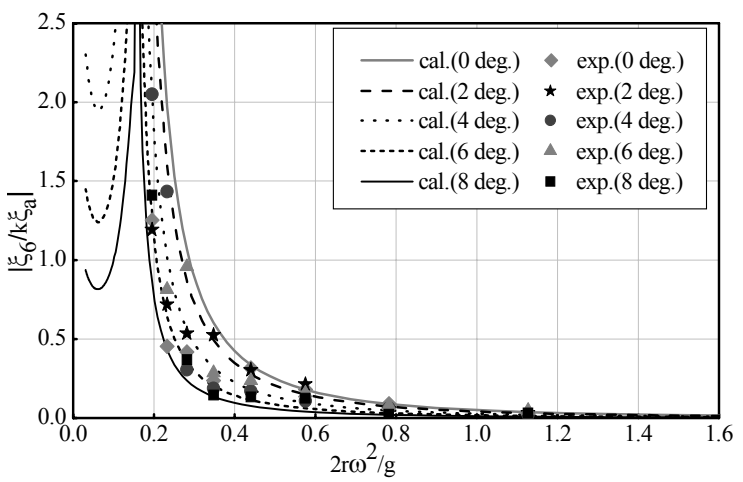

(d) Yaw

Fig. 13: RAO of (a) Surge; (b) Heave (c) Pitch; (d) Yaw motion at the angle of inclination

Figure 13 shows the experimental results for the RAOs of surge, heave, pitch and yaw motion and the comparisons with the numerical simulation using potential theory. As can be seen, the RAOs of surge, heave and pitch motion are found to yield an identical response throughout the frequencies in the numerical calculation with respect to the change of angle of inclination. But it can be seen that the angle of inclination only has an effect on yaw motion. The RAO of yaw motion is smaller at angles of inclination, compared to the upright condition, which is confirmed numerically and experimentally. It can be attributed to that the moment of inertia 
N.Mostafa, M. Murai, R.Nishimura, O. Fujita and Y. Nihei/ Journal of Naval Architecture and Marine Engineering 9(2012) 67-79 around the $w$ axis increases with increasing the angle of inclination (see Table 4), which is obtained with Eq. (13).

Figure 14 illustrates the results of RAO of yaw motion with the change of angle of inclination at normalized frequencies, $2 r \omega^{2} / g=0.44,0.57,0.78$ and 1.13 . This figure demonstrates an approximately linear relationship, where the RAO of yaw motion is reduced with increasing the angle of inclination. On the other hand, the yaw response is higher at lower frequencies. So, it is concluded that the effect of the angle of inclination is apparent at lower frequencies.

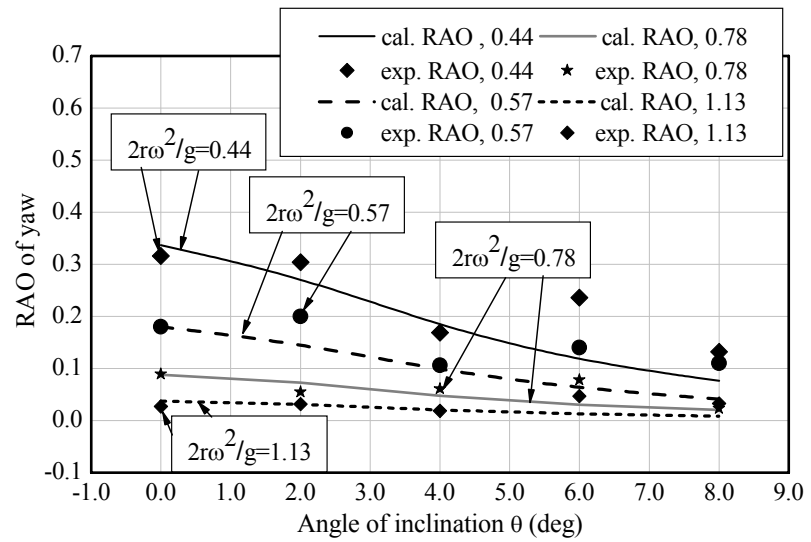

Fig. 14: Relationship between the RAO of yaw motion and the angle of inclination for normalized frequencies, $2 r \omega^{2} / g=0.44,0.57,0.78$, and 1.13

\section{Conclusions}

The floating wind turbine is a new technology in the offshore industry. In marine environment, the FOWT faces many problems, e.g. it can be inclined by the wind. Hence, in order to estimate motion characteristics of its actual model at angle of inclination, model test in regular waves were performed in a water tank at Yokohama National University. The experimental results have been compared with the numerical results and the following conclusion can be drawn:

i) The computed response of motion of a FOWT using potential theory exhibits a good agreement with the experimental results above the normalize frequency of 0.28 .

ii) Gyro moment caused by rotation of the FOWT blades has an effect on yaw motion, which is confirmed by numerical and experimental results.

iii) Variation of response of motion of a FOWT at angles of inclination is apparent in the yaw motion both numerically and experimentally. The RAO of yaw is reduced at inclined positions.

This experimental and numerical study indicates that for the actual prototype model, the yaw motion could be reduced at angle of inclination. These results could provide valuable information for the design of FOWT to extract the power from open sea.

\section{References}

Butterfield, S. Musial, W., Jonkman, J. and Sclavounos, P., (2005): Engineering challenges for floating offshore wind turbines, Copenhagen Offshore Wind Conference, Copenhagen, Denmark, October 26-28.

Fukumoto, Y., Moriya, Y., Tsuchiya, K., Hara, M. and Suzuki, H., (2006): A study of floating offshore wind turbine systems. Part III Investigation of reinforced concrete spar-buoy for offshore wind turbine, Proceedings of 28th Wind Energy Utilization Symposium, Tokyo, pp. 204-207.

Hurst, T. (2009): StatoilHydro Launches First Full Scale Floating Wind-Turbine, http://crispgreen.com/2009/09/statoilhydro-launches-first-full-scale-floating-wind-turbine/

Musial, W., Butterfield, S. and Boone, A (2004): Feasibility of floating platform system for wind turbines, The 23rd ASME Wind Energy Symposium, Reno, Nevada, January 5-8.

Murai, M. and Nishimura, R. (2010): A study on an experiment of behavior of a SPAR type offshore wind turbine considering rotation of wind turbine blades, Oceans 2010, Sydney, May 24-27. 
N.Mostafa, M. Murai, R.Nishimura, O. Fujita and Y. Nihei/ Journal of Naval Architecture and Marine Engineering 9(2012) 67-79

Nielsen, FG., Hanson, TD. and Skaare, B. (2006): Integrated dynamic analysis of floating offshore wind turbines, Proceeding of 25th International Conference on Offshore Mechanics and Arctic Engineering, Hamburg, Germany, OMAE2006-92291.

Roddier, D., Cermelli, C. and Weinstein, A. (2010): Wind float: a floating foundation for offshore wind turbines, Journal of Renewable and Sustainable Energy, vol. 2(3), 2010, pp. 033104-1 34. doi:10.1063/1.3435339

Skaare, B., Hanson, T. D., Nielsen, F. G., Yttervik, R., Hansen, A. M., Thomsen, K and Larsen, T. J. (2007): Integrated dynamic analysis of floating offshore wind turbine, European Wind Energy Conference and Exhibition, Milan, May 7-10.

Suzuki, H. and Sato, A. (2007): Load on turbine blade induced by motion of floating platform and design requirement for the platform, Proceedings of 26th International Conference on Offshore Mechanics and Arctic Engineering, California, USA, OMAE2007-29500.

Suzuki, H. and Ashida, T. (2005): Optimization of floating structure of a spar-type floating wind turbine, Proceedings of JASNAOE 2005S, pp.107-108.

Utsunomiya, T. and Nishida, E. (2010): On sea experiment of a hybrid SPAR for floating offshore wind turbine 1/10 scale model, Proceedings of the ASME 29th International Conference on Ocean, Offshore and Arctic Engineering, Shanghai, China, OMAE2010-20730. 